\title{
Three-Dimensional Surface Evaluation and Shear Bond Strength of Three Pre-treatment E-max Surfaces for Metal and Ceramic Orthodontic Brackets (In Vitro Study)
}

\author{
Huda A. Mohammed*, Omar S. Ali \\ Department of Orthodontic, Faculty of Dentistry, Tishk International University, Erbil, Kurdistan Region, Iraq
}

\section{*Corresponding author: \\ Huda A. Mohammed, \\ Department of Orthodontic, \\ Faculty of Dentistry, Tishk International University, Erbil, \\ Kurdistan Region, Iraq. \\ E-mail: hudaadelmohammed @gmail.com}

Received: 29 March 2020

Accepted: 10 June 2020

Published: 30 December

2020

DOI

10.25156/pti.v10n2y2020.pp113-118

\section{A B S T R A C T}

3D evaluation and shear bond strength (SBS) for metal and ceramic brackets after three pre-treatment surfaces of lithium disilicate (LD) crowns. Sixty lithium disilicate (LD) crowns were fabricated in laboratory according to manufacturer instruction and then divided into six groups (three groups for metal brackets and three groups for ceramic one). The $1^{\text {st }}$ group for both was treated with round diamond bur, the $2^{\text {nd }}$ one by hydrofluoric acid (HFA) $9.0 \%$, and the $3^{\text {rd }}$ group by laser (Er,Cr:YSGG). All treated surfaces were examined by laser profilometer and scan electron microscope. The Scotchbond Universal Adhesive and Transbond XT were used for bracketing procedure for metal and ceramic bracket (central incisor, 0,022" slot). Universal testing machine was used for shear bond. A crosshead speed of $1 \mathrm{~mm} / \mathrm{min}$ was used, and the maximum load necessary to deboned the bracket will be recorded. HFA treated surfaces gave a highest SBS for both metal and ceramic brackets, then laser and finally the bur. The lowest roughness parameter Sa (arithmetical mean height) was in bur, both laser and HFA gave no significant differences. The HFA gave a highest $\mathrm{Sz}$ (maximum height) and Spc (arithmetic mean peak curvature) value. To increase the shear bond strength, the HFA is one of the best methods for roughness although the laser gave nearby roughness parameters. Ceramic brackets gave higher SBS than metal one with all pre-treatment procedures.

Keywords: Ceramic orthodontic brackets; Laser profilometer; Lithium disilicate crowns; Roughness; Shear bond strength

\section{INTRODUCTION}

In the past years, demand for orthodontic care has increased considerably in adult patients and many of these patients have ceramic restorations, direct and indirect one such as veneer and crowns. E max has achieved significant renown due to marked progress in the ability to imitate dental tissues, mechanical strength (Sundfeld et al., 2016). However, bonding of orthodontic bracket to ceramic surface requires special care because they can show a higher degree of failure in relation to enamel bond (Matos et al., 2016). At the other hand, some experiments did not show any adverse effects on the ceramic surface, as the most common failures were adhesive between the bonding material and the ceramic surface (Costa et al., 2012, Abreu et al., 2015, Costa et al., 2015).

Lithium disilicate crown (LDC) has gained popularity over the time due to better esthetics, biocompatibility, and translucency (Albakry et al., 2004, Kuijper et al., 2019). To improve shear bond strength, change in surface characteristics of $\mathrm{LDC}$ is proposed before bracket bonding.
Available literature highlights different approaches which can either be chemical, mechanical, or combination of both for surface treatment of LDC (Türk et al., 2006).

Grinding of the LD crowns surfaces with diamond bur, sandpaper disc, or blasting with $\mathrm{Al} 2 \mathrm{O} 3$ abrasives is mechanical approach which used for roughening the LDC surfaces (Gillis and Redlich, 1998). However, these procedures produced a permanently destructive effect. The chemical approaches entail acid etching to provide bonding to adhesive materials to adhere to ceramic restoration (Nagayassu et al., 2006). Furthermore, one of the researches was used different concentration between $9.5 \%$ and $10 \%$ hydrofluoric acid (HFA) at different contact time to examine the capability for creating irregularities on the ceramic surface (Barbosa et al., 1995, Canay et al., 2001, Özcan et al., 2001, Harari et al., 2003).

Lasers are another alternative ways for roughness and many types of laser were examined shear bond strength (SBS) of brackets with different types of esthetic crowns such as Er:YAG (erbium-doped yttrium aluminum garnet 
laser, erbium YAG laser), Nd:YAG (neodymium-doped yttrium aluminum garnet laser), Er,Cr:YSGG (erbium, chromium-doped yttrium, scandium, gallium, and garnet laser), and $\mathrm{CO}_{2}$ (carbon dioxide laser) (Basaran et al., 2007, Von Fraunhofer et al., 1993).

The limitation of researches and data for this subject and difficulty to control between high bonding with less damaging of surface, with increasing the demand from clinical view, the $3 \mathrm{D}$ analysis was done.

\section{MATERIALS AND METHODS}

\section{Sample Preparation}

An intact central incisor that was extracted for periodontal reasons was used in the present study. Tooth was scaled with ultrasonic scaler to remove tissue tags and plaque kept in water at $4{ }^{\circ} \mathrm{C}$ with addition of $0.5 \%$ sodium azide, then the tooth was prepared for LD crown preparation. The prepared tooth was duplicated to 60 teeth to be as standardized criteria. All impression (heavy and light body) for duplication was poured by a high stone strength. After that the duplication of the prepared tooth with additional vulcanizing duplication silicone was ready to pour with pattern resin to be strong enough like a tooth structure under crown and SBS test.. The laboratory work was start to fabricate LDC according to material requirements. The teeth were randomly divided to two groups: Group (Ia): $(n=30)$ Crowns for bonding a stainless steel brackets and Group (Ib): $(n=30)$ Crowns for bonding a ceramic bracket.

These groups were subdivided into three subgroups depending on treatment surface of both types of crowns (Area for treatment about $[4.50 \mathrm{~mm} \times 3.50 \mathrm{~mm}]$ ) to accommodate the bracket as follows:

Group (Ia, b-1, $n=20)$ : The surface was treated by HFA $4.5 \%$ (Ultradent, USA). Group (Ia, b-2, $n=20$ ): The surface will be treated by laser (erbium, chromium:yttrium, scandium, gallium, garnet) (Waterlase iPlus, USA). Group (Ia, b-3, $n=20)$ : The surface will be treated by bur, Figure 1 .

All crowns were mounted horizontally in self-cure acrylic resin that only buccal surface of the crown at a level slightly below cement enamel junction will be exposed. To ensure the parallelism of labial aspect of crowns, dental surveyor (Dentalfarm-Torino-Italy) was used.

\section{Surface Preparation}

Different surface treatments were done before bonding procedure, the treatment areas were drawn at labial surfaces and then specimens were divided into the following groups:

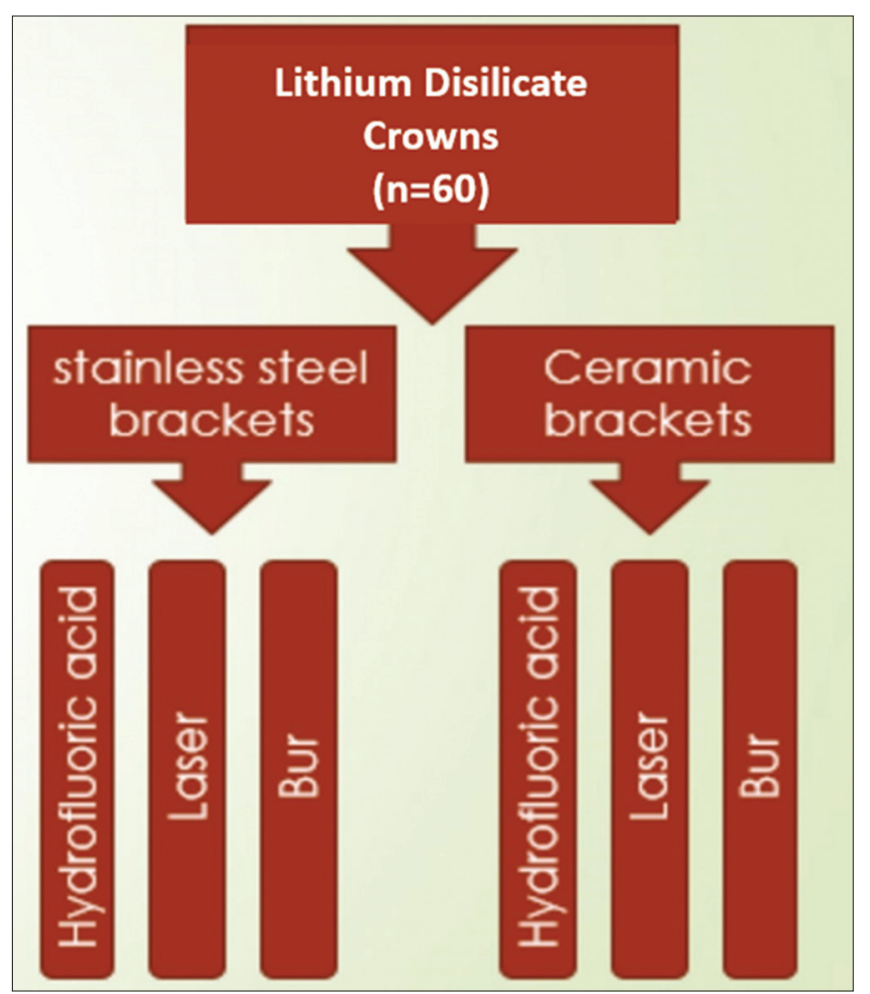

Figure 1: Diagram of the study sample classification

1. Group (Ia, b-1): HFA was used as $4.5 \%$ concentration for $120 \mathrm{~s}$, then rinsed with a water for $30 \mathrm{~s}$ and dry with normal dental air syringe according to manufacturer instruction of acid

2. Group (Ia, b-2): Laser (Er,Cr:YSGG) was used for etching the selected area as following (MX5, 6W, 10 water, 70 air, $50 \mathrm{~Hz}$ ): Surfaces were etched very slowly without touching with fixed distance $(2 \mathrm{~mm})$ through holding of hand of the laser with holder of the crowns

3. Group (Ia, b-3): Roughness of selected area by round diamond bur (Shofu, Japan) through holding of turbine by holder and apply simple touching of crown in selected area.

\section{Roughness of Surfaces}

Roughness of pretreated areas was examined by laser microscope profilometer (KENYNCE, VK 1100, USA). The device automatically focuses at the area that you need to examined. 3D images and the surface roughness parameters $\mathrm{Sa}$ (average roughness), Spc (arithmetic mean peak curvature), and $\mathrm{Sz}$ (average peak to valley high depths of five consecutive sampling measurements) were examined.

\section{Scanning Electron Microscope (SEM)}

The pre-treatment area of crowns was sputter coated with a thin carbon layer and then examined by SEM (Tescan Mira3, USA) under the following conditions, high vacuum, 5.0 KV accelerating voltage), WD $30.19 \mathrm{Nm}$ at different 
magnification $\times 28, \times 100, \times 500$, and $\times 1000$. SEM was examined at TU Bergakademie Freiberg/Germany.

\section{Bonding of Brackets}

After all the surface treatment, a silane coupling agent (Monobond Plus ceramic primer Ivoclar Vivadent) was applied to the etched ceramic surface of all the groups by micro-brush in thin layer for $60 \mathrm{~s}$ and air dried. Sixty central incisor metal and ceramic orthodontic brackets were used (Roth, 0.022-in slot; 3M Unitek, Monrovia, California), after that a small amount of adhesive (Transbond XT, $3 \mathrm{M}$, USA) was placed on the meshed area of the brackets in all samples then the bracket was transfer using bracket holder tweezer (Dentaurum, Germany) toward the bonded surface of the tooth then its position in occlusogingival direction is adjusted with bracket positioning gauge after that pushed firmly with the aid of flat end rod of surveyor and with load mass of $300 \mathrm{~g}$ for $10 \mathrm{~s}$ (Rêgo and Roman, 2007). The excess of the resin was removed by probe, adhesive was cured with light cure device (Elipar, 3M, USA) with 5 $\mathrm{mm}$ away from the bracket through special metal holder fixed on square metal base for $40 \mathrm{~s}$ (10 s for each side; cervical, incisal, mesial, and distal surface) at an intensity of $1600 \mathrm{~mW} / \mathrm{cm}^{2}$ (Attin et al., 2012). The intensity of light cure was checked before each curing. Once bracket bonding completed, the specimens were stored in normal saline inside incubator at $37^{\circ} \mathrm{C}$ for 1 day.

\section{SBS Test}

All the samples were placed in special holder connected to the base plate of universal testing machine (GOTECH). A chisel-edge plunger was mounted in the movable crosshead of the testing machine. The position of chisel edge was directed toward so that the leading bracket-tooth interface parallel to the labial tooth surface. A crosshead speed of $1 \mathrm{~mm} / \mathrm{min}$ was used, and the maximum load necessary to deboned the brackets were recorded as described by Trites et al. (2004). The bond failure load was recorded using a special software in PC connected to the testing machine. The load failure was recorded in Newton $(\mathrm{N})$ and the stress was calculated in mega Pascal $\left(1 \mathrm{MPa}=\mathrm{N} / \mathrm{mm}^{2}\right)$ by dividing the force in Newton by the area of the bracket base. The digital caliper was used for measuring the base (width, length) of both brackets. SBS test was done in Salahaddin University: College of Engineering - Department of Mechanical Engineering, Figure 2.

\section{Statistical Analysis}

One-way ANOVA plus Holm-Sidak test (roughness) and Kruskal-Wallis one-way ANOVA on ranks plus Tukey test (shear bond strength) were used to evaluate statistically significant differences at a 95\% confidence level. Statistical analysis will perform by SPSS 17.0 Software (SPSS Inc., Chicago, USA).

\section{RESULTS}

\section{Roughness}

The 3D images of pretreated areas are depicted in Figure 3. Roughness parameters showed that highest $S$ a value gave by $\mathrm{L}$ and HA more that $\mathrm{B}$. The HFA gave also higher $\mathrm{Sz}$ value comparing with others. The all parameters of roughness are shown in Table 1.

\section{SEM}

The images from SEM showed different morphological feature started from low magnification to high magnification of pre-treatment surfaces of LD crowns.

\section{Pre-treatment surfaces by bur (B)}

At low magnification $\times 28$, the images showed different lines like with crossing with others due to the lines of bur during roughness procedure comparing with the

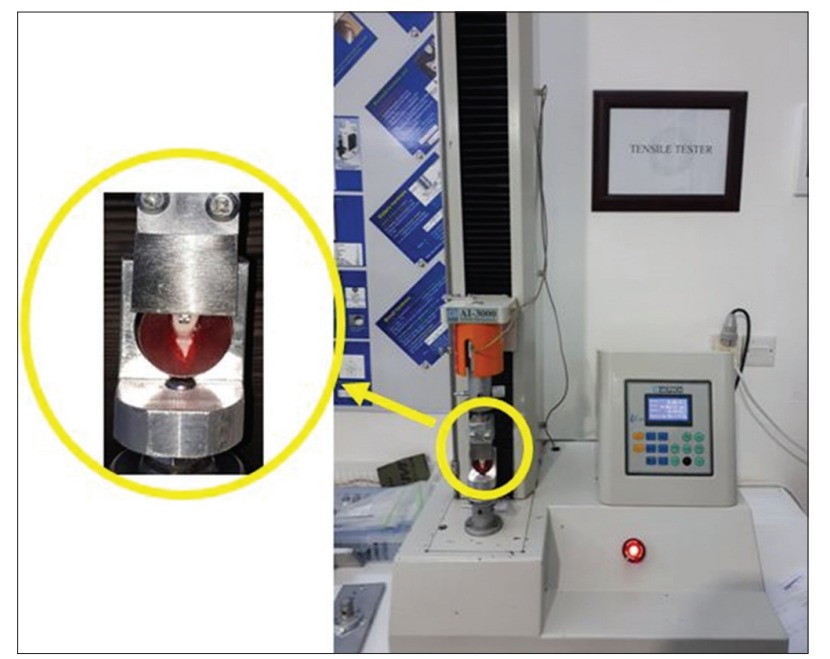

Figure 2: Universal testing machine GOTECH (shear bond strength)

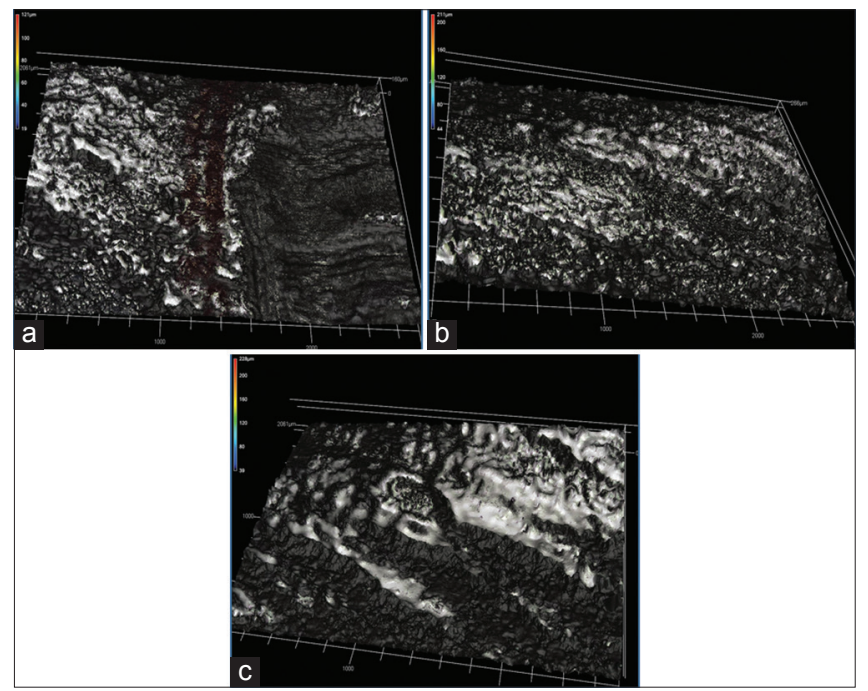

Figure 3: 3D profilometer images of pre-treatment of LDC. (a) By bur. (b) By hydrophilic acid. (c) By laser 
surrounding normal areas that not touch it by bur. During increasing of magnification to higher one $(\times 100$ and $\times 500$ ), the differences between two areas appeared more clear, normal crown appeared more uniform surfaces with certain protrusion groups spread it randomly comparing with brackets areas that appeared more abraded areas with protrusions / recession, Figure 4.

\section{Pre-treatment surfaces by HFA}

At low magnification $\times 28$, the images showed more uniform surfaces in both etched and non-etched areas, with increase the magnification from $\times 100, \times 500$, until $\times 1000$, the images of pretreated areas showed increase the numbers of dark hole like with different sizes spread it randomly with uniform rough like structures with some elevation. The untouched areas appeared more uniform with very little dark hole like, Figure 5.

\section{Pre-treatment with laser (L)}

At low magnification $\times 28$, the surfaces appeared more uniform. When increase magnification, the images showed white lines and point explained that these areas have some elevation randomly spread it, Figure 6.

\section{SBS}

The HFA treated crowns gave a higher SBS of both metallic and ceramic brackets and the treated crowns by $\mathrm{B}$ gave a

Table 1: Results of roughness (means and standard deviations and ANOVA test)*

\begin{tabular}{lccc}
\hline Process & Sa $(\mu \mathrm{m})$ & $\mathrm{Sz}(\mu \mathrm{m})$ & Spc $(\mu \mathrm{m})$ \\
\hline Bur & $9.87(0.32)^{\mathrm{a}}$ & $105.75(2.1)^{\mathrm{c}}$ & $1067.53(0.11)^{\mathrm{b}}$ \\
Hydrofluoric acid & $10.32(1.22)^{\mathrm{b}}$ & $141.32(066)^{\mathrm{a}}$ & $1365.93(1.4)^{\mathrm{c}}$ \\
Laser & $10.81(0.5)^{\mathrm{b}}$ & $131.07(0.32)^{\mathrm{b}}$ & $927.89(1.55)^{\mathrm{a}}$ \\
\hline
\end{tabular}

*Same superscripts per column imply mean values with no statistically significant difference ( $P>0.05)$
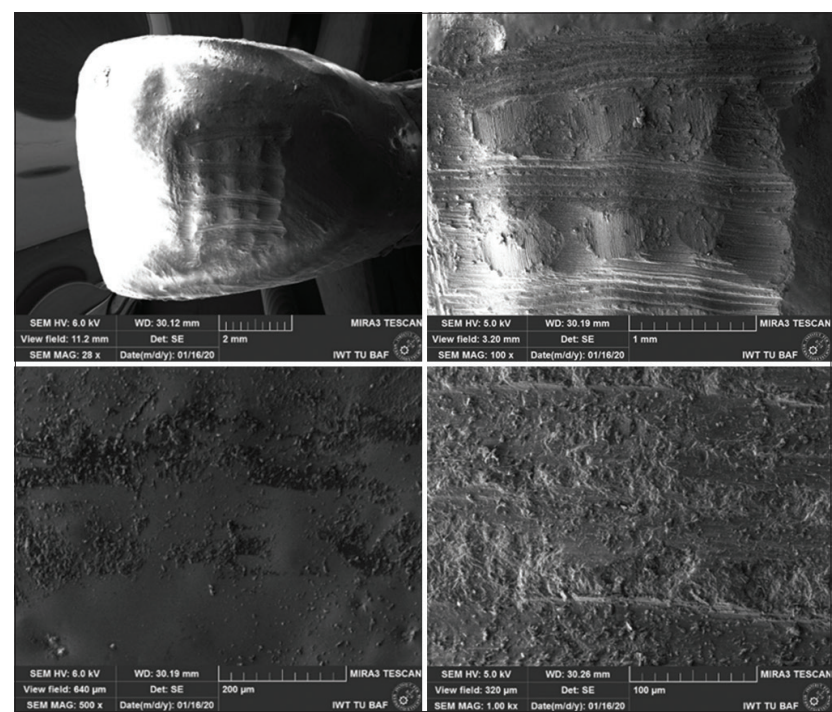

Figure 4: Scanning electron microscope images of pretreatment surfaces by bur at $\times 28, \times 100, \times 500$, and $\times 1000$ lowest one. The treated crowns by $\mathrm{L}$ gave between both HFA and B. The ceramic brackets as total gave a higher SBS than metallic one as shown in Table 2.

\section{DISCUSSION}

The main factor affecting the success of orthodontic treatment is how to keep the brackets adhere to tooth structure and decrease the rate of debonding. Many researches focusing to increase the SBS of brackets to tooth structure ('Trakyali et al., 2009, Al-Hity et al., 2012, Faltermeier et al., 2013, Grewal Bach et al., 2014).

Nowadays, many patients seeking for orthodontic treatment and they have a LD crowns or veneers as one

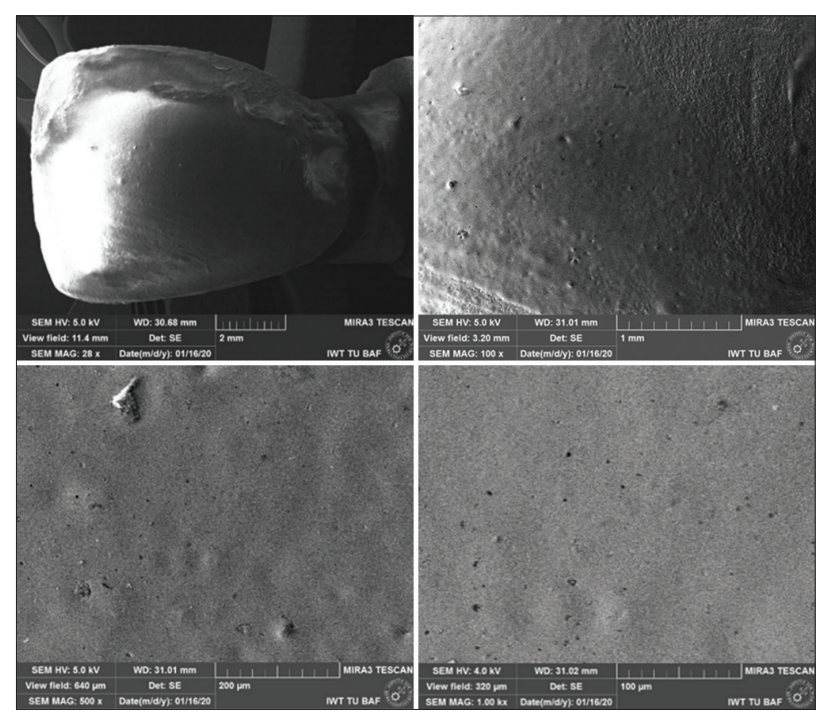

Figure 5: Scanning electron microscope images of pre-treatment surfaces by hydrofluoric acid at $\times 28, \times 100, \times 500$, and $\times 1000$

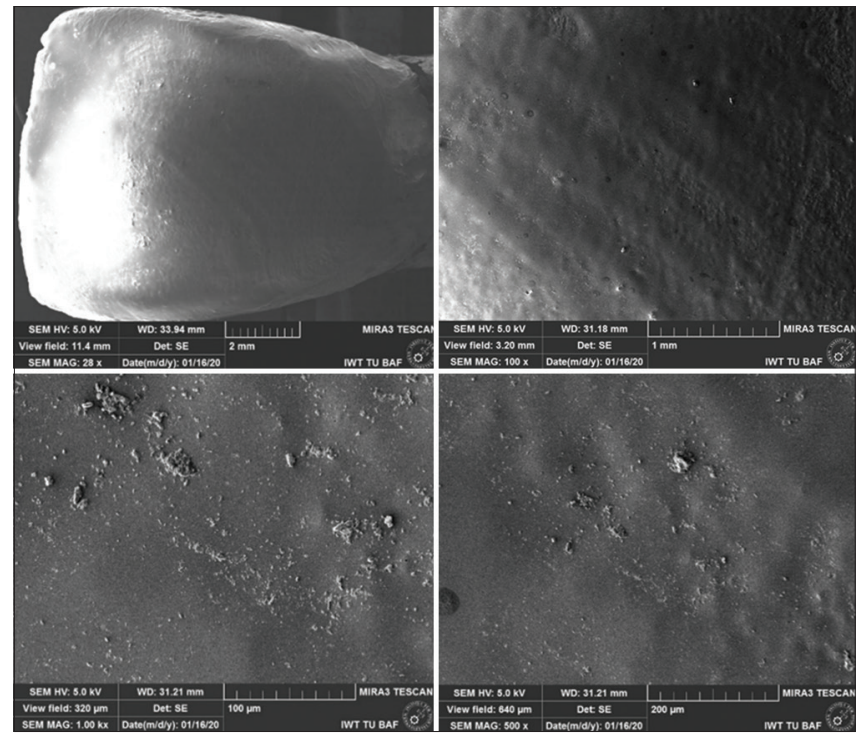

Figure 6: Scanning electron microscope images of pre-treatment surfaces by laser at $\times 28, \times 100, \times 500$, and $\times 1000$ 
Table 2: Results of SBS (means and standard deviations)*

\begin{tabular}{lcc}
\hline Process & Metallic brackets & Ceramic brackets \\
\hline Bur & $10.85(0.44)^{\mathrm{c}}$ & $16.22(0.22)^{\mathrm{c}}$ \\
Hydrofluoric acid & $15.45(1.2)^{\mathrm{a}}$ & $19.46(2.1)^{\mathrm{a}}$ \\
Laser & $12.80(0.76)^{\mathrm{b}}$ & $17.5(0.67)^{\mathrm{b}}$ \\
\hline
\end{tabular}

*Same superscripts per column imply mean values with no statistically significant difference $(P>0.05)$

of the esthetic treatments. LD crowns were introduced as machinable materials to respond to the demanded increased strength, toughness, and wear resistance, required for the fabrication of dental pieces (Monmaturapoj et al., 2013, Denry and Holloway, 2010).

The present study showed that the ceramic brackets as total gave more SBS than metallic brackets and this results agree with many studies due to a stronger adhesion to ceramics and light transmission, which leads to a higher degree of polymerization and stress reduction on the adhesive bracket joint (Zachrisson et al., 1996, Abu Alhaija et al., 2010, Girish et al., 2012).

It has been suggested that clinically adequate bond strength for orthodontic brackets to enamel should be from 6 to 8 MPa (Endo et al., 2008).

The mean SBS of both metallic and ceramic brackets to LD crown achieved in this study fell within this range or exceeded this limit, and therefore, from mechanical point of view, the three treated methods could be considered sufficient in bonding strength between the orthodontic bracket and the LD crown in clinical applications (Türk et al., 2006).

HFA acid exhibited high SBS this could be explained by the ability of HF acids to attack the glassy phase of the ceramic, dissolving the surface to the depth of a few micrometers, and consequently, a LD crystal was protruded from the glassy matrix (Prochnow et al., 2017), the resultant altered topography increased the surface area for micromechanical bonding with resin composites (Ramakrishnaiah et al., 2016).

The present study showed that SBS for the mechanical roughness with bur for LD crown is lower than HFA and this disagree with Schmage et al. (2003) that showed roughening the ceramic surfaces with either diamond bur or sandblasting created higher surface roughness than HF acid etching or silicatization.

In this study, the laser process actually produced a high bond strength for LD crown, however, no crazing effect was generated on the ceramic after laser lasing and debonding. This contrast with other studies that indicated laser treated ceramic did not enhance adhesion with resin cement (Erdem et al., 2014). This is probably associated with the differences in ceramic materials and the method of laser treating procedure.

\section{CONCLUSION}

1. The ceramic brackets gave highest SBS comparing with metallic one

2. Hydrofluoric acid gave a highest SBS comparing with laser and bur in both metallic and ceramic brackets

3. Hydrofluoric acid gave a highest roughness parameter comparing with bur and laser

4. The lowest value of roughness and SBS was in bur pretreatment procedure

5. The results of three methods of pretreatment are efficient clinically

6. Differences in surface roughness were found after pretreatment methods and this result improving in SBS in three methods

7. The morphology of the pre-treatment surfaces presented a variety of topographical characteristics representing in images of SEM.

\section{REFERENCES}

Abreu, H. F., A. R. Costa, A. B. Correr, S. A. Vedovello, H. C. Valdrighi and E. C. Santos. 2015. Influence of light source, thermocycling and silane on the shear bond strength of metallic brackets to ceramic. Braz. Dent. J. 26(6): 685-688.

Abu Alhaija, E. S. A., I. A. A. AlReesh and A. M. AlWahadni. 2010. Factors affecting the shear bond strength of metal and ceramic brackets bonded to different ceramic surfaces. Eur. J. Orthod. 32(3): 274-280.

Albakry, M., M. Guazzato and M. V. Swain. 2004. Effect of sandblasting, grinding, polishing and glazing on the flexural strength of two pressable all-ceramic dental materials. J. Dent. 32(2): 91-99.

Al-Hity, R., M. P. Gustin, N. Bridel, L. Morgon and B. Grosgogeat. 2012. In vitro orthodontic bracket bonding to porcelain. Eur. J. Orthod. 34(4): 505-511.

Attin, R., B. Stawarczyk, D. Keçik, M. Knösel, D. Wiechmann and T. Attin. 2012. Shear bond strength of brackets to demineralize enamel after different pretreatment methods. Angle Orthod. 82(1): 56-61.

Barbosa, V. L., M. A. Almeida, O. Chevitarese and O. Keith. 1995. Direct bonding to porcelain. Am. J. Orthod. Dentofacial Orthop. 107: 159-164.

Basaran, G., T. Özer, N. Berk and O. Hamamci. 2007. Etching enamel for orthodontics with an erbium, chromium: Yttrium-scandiumgallium-garnet laser system. Angle Orthod. 77(1): 117-123.

Canay, S., N. Hersek and A. Ertan. 2001. Effect of different acid treatments on a porcelain surface. J. Oral Rehabil. 28: 95-101.

Costa, A. R., A. B. Correr, R. M. Puppin-Rontani, S. A. Vedovello, H. C. Valdrighi and L. Correr-Sobrinho. 2012. Effect of bonding material, etching time and silane on the bond strength of metallic orthodontic brackets to ceramic. Braz. Dent. J. 23(3): 223-227.

Costa, A. R., A. B. Correr, S. Consani, M. C. Giorgi, S. A. Vedovello and M. F Vedovello. 2015. Influence of water storage and bonding material on bond strength of metallic brackets to ceramic. Braz. 
Dent. J. 26(5): 503-506.

Denry, I. and J. A. Holloway. 2010. Ceramics for dental applications: A review. Materials. 3(1): 351-368.

Endo, T., R. Ozoe, K. Shinkai, J. Shimomura, Y. Katoh and S. Shimooka. 2008. Comparison of shear bond strengths of orthodontic brackets bonded to deciduous and permanent teeth. Am. J. Orthod. Dentofacial. Orthop. 134: 198-202.

Erdem, A., G. C. Akar, A. Erdem and T. Kose. 2014. Effects of different surface treatments on bond strength between resin cements and zirconia ceramics. Oper. Dent. 39: E118-E127.

Faltermeier, A., C. Reicheneder, P. Götzfried and P. Proff. 2013. Bonding orthodontic ceramic brackets to ceramic restorations: Evaluation of different surface conditioning methods. MSA. 4(7): 10-14.

Gillis, I. and M. Redlich. 1998. The effect of different porcelain conditioning techniques on shear bond strength of stainless steel brackets. Am. J. Orthod. Dentofacial. Orthop. 114: 387-392.

Girish, P. V., U. Dinesh, C. Bhat and P. C. Shetty. 2012. Comparison of shear bond strength of metal brackets bonded to porcelain surface using different surface conditioning methods: An in vitro study. J. Contemp. Dent. Pract. 13(4): 487-493.

Grewal Bach, G. K., Y. Torrealbab and M. O. Lagravère. 2014. Orthodontic bonding to porcelain a systematic review. Angle Orthod. 84(3): 555-560.

Harari, D., S. Shapira-Davis, I. Gillis, I. Roman and M. Redlich. 2003. Tensile bond strength of ceramic brackets bonded to porcelain facets. Am. J. Orthod. Dentofacial. Orthop. 123: 551-554.

Kuijper, M., M. Gresnigt, M. van den Houten, D. Haumahu, U. Schepke and M. CuneFracture. 2019. Fracture strength of various types of large direct composite and indirect glass ceramic restorations. Oper. Dent. 44(4): 433-442.

Matos, N. R., A. R. Costa, H. C. Valdrighi, A. B. Correr, S. A. Vedovello and M. Jr. Santamaria. 2016. Effect of acid etching, silane and thermal cycling on the bond strength of metallic brackets to ceramic. Braz. Dent. J. 27(6): 734-738.

Monmaturapoj, N., P. Lawita and W. Thepsuwan. 2013. Characterisation and properties of lithium disilicate glass ceramics in the SiO2-Li2O-K2O-Al2O3 system for dental applications. Adv. Mater. Sci. Eng. 2013: 1.

Nagayassu, M. P., L. K. Shintome, E. S. Uemura, J. E. Araújo. 2006.
Effect of surface treatment on the shear bond strength of a resinbased cement to porcelain. Braz. Dent. J. 17: 290-295.

Özcan, M., H. N. Alkumru and D. Gemalmaz. 2001. The effect of surface treatment on the shear bond strength of luting cement to a glassinfiltrated alumina ceramic. Int. J. Prosthodont. 14: 335-339.

Prochnow, C., A. B. Venturini, R. Grasel, M. C. Bottino and L. F.VaLandro. 2017. Effect of etching with distinct hydrofluoric acid concentrations on the flexural strength of a lithium disilicatebased glass ceramic. J. Biomed. Mater. Res. B Appl. Biomater. 105: 885-891.

Ramakrishnaiah, R., A. Alkheraif, D. Divakar, J. P. Matinlinna and P. K. Vallittu. 2016. The effect of Hydrofluoric acid etching duration on the surface micromorphology, roughness, and wettability of dental ceramics. Int. J. Mol. Sci. 17: 822.

Rêgo, E. B. and F. L. Roman. 2007. Shear bond strength of metallic brakets photo-activated with light-emitting diode (led) at different exposure time. J. Appl. Oral Sci. 15: 412-415.

Schmage. P., I. Nergiz, W. Herrmann and M. Özcan. 2003. Influence of various surface-conditioning methods on the bond strength of metal brackets to ceramic surfaces. Am. J. Orthod. Dentofacial. Orthop. 123: 540-546.

Sundfeld, D., L. Correr-Sobrinho, N. I. P. Pini, A. R. Costa, R. H. Sundfeld and C. S. Pfeifer. 2016. Heat treatment-improved bond strength of resin cement to lithium disilicate dental glassceramic. Ceram Int. 42(8): 10071-10078.

Trakyali, G., Ö. Malkondu, E. Kazazoğlu and T. Arun. 2009. Effects of different silanes and acid concentrations on bond strength of brackets to porcelain surfaces. Eur. J. Orthod. 31(4): 402-406.

Trites, B., T. F. Foley and D. Banting. 2004. Bond strength comparison of 2 self-etching primers over month storage period. Am. J. Orthod. Dentofacial. Orthop. 126: 709-716.

Türk, T., D. Saraç, Y. S. Saraç and S. Elekdağ-Türk. 2006. Effects of surface conditioning on bond strength of metal brackets to allceramic surfaces Eur. J. Orthod. 28: 450-456.

Von Fraunhofer, J. A., D. J. Allen and G. M. Orbell. 1993. Laser etching of enamel for direct bonding. Angle Orthod. 63(1): 73-76.

Zachrisson, Y. Ø., B. U. Zachrisson and T. Büyükyilmaz. 1996. Surface preparation for orthodontic bonding to porcelain. Am. J. Orthod. Dentofacial Orthop. 109(4): 420-430. 